\title{
Mechanisms that could increase cancer vulnerability in COVID-19 mRNA vaccine recipients
}

\begin{abstract}
The BNT162b2 and mRNA-1273 vaccines have been rolled out globally in high numbers. Even minimal risk groups have been fully vaccinated in many countries. We observe certain links that show there are ways cancer can have an easier path to establishing itself as a result of COVID-19 mRNA vaccination.
\end{abstract}

\section{Dysregulation by spike protein}

Proteins p53 and BRCA1 are well-known tumor suppressor proteins that regulate downstream genes in response to numerous cellular stresses and are frequently mutated in human cancer ["p53"][Silver, Daniel P., and David M. Livingston]. It is observed to be inhibited by SARS-CoV-2 [Stingi, Aureliano, and Lu.]. The S2 (Spike protein S2) subunit of Sars-CoV-2 has known interactions with p53, but also with BRCA-1/2 proteins [Singh, Nishant, and Anuradha Bharara Singh.]. That could also mean that the proteins generated by the mRNA vaccines would also interact with the p53 gene, and inhibit this tumor suppressor. The SARS-CoV spike protein is also known to cause apoptosis in Vero E6 cells. Cleavage of the $\mathrm{S}$ protein into fragments was suggested in previous studies, which includes a form that resembles the $S 2$ protein in this study, suggesting the spike proteins are enough to degrade p53 [Chow, K. Y., et al.] [Barhoumi, Tlili, et al.]. Even though vaccines help prevent SARS-CoV-2 spread and infection, individuals with solely vaccine-gained immunity might suffer from opsonization on initial infection, eventually resulting in high viral load and spike overload [Bahnan, Wael, et al.] [Acharya, Charlotte B., et al.]. It is important to note that certain results suggest that recombinant spike-based SARS-CoV-2 immunogen glycosylation reproducibly recapitulates signatures of viral glycosylation. [Allen, Joel D., et al.]. Which could potentially be cancer-enhancing [Kremsreiter, Stefanie Maria, et al.], even though this does not seem like a very likely scenario, it would probably increase the chances of p53 being inhibited [May, and May].

\section{Code issues in mRNA}

Several natural mechanisms of termination suppression exist, including ribosomal frameshifting. The process of protein synthesis termination, although effective, is not $100 \%$ efficient [Dabrowski, Maciej, et al.]. Several +1 frameshift sites have also been recognized in eukaryotic mRNA. For example, the expression of mammalian antizyme 1 (AZ1) requires a +1 frameshift [Moon, et al.]. Codon misreading tRNAs are known to promote tumor growth in mice [Santos, Mafalda, et al.], and it's known that many human cancers can be caused by illogical frameshifts [Lee, Hui-Ling Rose, \& Joseph Dougherty.] [X; Xia.]. In BNT162b2 and mRNA-1273 vaccines there is a relatively high chance of such occurrences due to poor choices of stop codons and shoddy optimization [Mordstein, Christine, et al.].

\section{Discussion}

Tumor suppressors are important in preventing cancer from occuring. Mass vaccination on low risk groups requires more data on this matter before determining the safety of the current mRNA vaccines. 


\section{References}

Acharya, Charlotte B., et al. No Significant Difference in Viral Load Between Vaccinated and Unvaccinated, Asymptomatic and Symptomatic Groups When Infected with SARS-CoV-2 Delta Variant. Cold Spring Harbor Laboratory, 29 Sept. 2021, http://dx.doi.org/10.1101/2021.09.28.21264262.

Allen, Joel D., et al. Site-Specific Steric Control of SARS-CoV-2 Spike Glycosylation. Cold Spring Harbor Laboratory, 9 Mar. 2021, http://dx.doi.org/10.1101/2021.03.08.433764.

Bahnan, Wael, et al. Opsonization by Non-Neutralizing Antibodies Can Confer Protection to SARS-CoV-2 despite Spike-Dependent Modulation of Phagocytosis. Cold Spring Harbor Laboratory, 15 Oct. 2021, http://dx.doi.org/10.1101/2021.10.14.464464.

Barhoumi, Tlili, et al. "SARS-CoV-2 Coronavirus Spike Protein-Induced Apoptosis, Inflammatory, and Oxidative Stress Responses in THP-1-Like-Macrophages: Potential Role of Angiotensin-Converting Enzyme Inhibitor (Perindopril)." Frontiers in Immunology, vol. 0, Jan. 2021, https://doi.org/10.3389/fimmu.2021.728896.

Chow, K. Y., et al. "SARS Coronavirus and Apoptosis - PubMed." Hong Kong Medical Journal = Xianggang Yi Xue Za Zhi, vol. 14 Suppl 4, Aug. 2008.

Dabrowski, Maciej, et al. “Translational Readthrough Potential of Natural Termination Codons in Eucaryotes--The Impact of RNA Sequence." RNA Biology, vol. 12, no. 9, 2015, pp. 950-58, https://doi.org/10.1080/15476286.2015.1068497.

Johnson, Philip L. F., et al. "Vaccination Alters the Balance between Protective Immunity, Exhaustion, Escape, and Death in Chronic Infections." Journal of Virology, vol. 85, no. 11, June 2011, pp. 5565-70, https://doi.org/10.1128/JVI.00166-11.

Kremsreiter, Stefanie Maria, et al. "Glycan-Lectin Interactions in Cancer and Viral Infections and How to Disrupt Them." International Journal of Molecular Sciences, vol. 22, no. 19, Sept. 2021, p. 10577, https://doi.org/10.3390/ijms221910577.

Lazzaro, Sandra, et al. "CD8 T-Cell Priming upon MRNA Vaccination Is Restricted to Bone-Marrow-Derived Antigen-Presenting Cells and May Involve Antigen Transfer from Myocytes." Immunology, vol. 146, no. 2, Oct. 2015, pp. 312-26, https://doi.org/10.1111/imm.12505.

Lee, Hui-Ling Rose, and Joseph P. Dougherty. "Pharmaceutical Therapies to Recode Nonsense Mutations in 
Inherited Diseases - PubMed." Pharmacology \& Therapeutics, vol. 136, no. 2, Nov. 2012,

https://doi.org/10.1016/j.pharmthera.2012.07.007.

May, and May. "Twenty Years of P53 Research: Structural and Functional Aspects of the P53 Protein."

Oncogene, vol. 18, no. 53, Dec. 1999, pp. 7621-36, https://doi.org/10.1038/sj.onc.1203285.

Moon, et al. "Predicting Genes Expressed via -1 and +1 Frameshifts." Nucleic Acids Research, vol. 32, no. 16,

Jan. 2004, pp. 4884-92, https://doi.org/10.1093/nar/gkh829.

Mordstein, Christine, et al. "Transcription, MRNA Export, and Immune Evasion Shape the Codon Usage of Viruses." Genome Biology and Evolution, vol. 13, no. 9, May 2021,

https://doi.org/10.1093/gbe/evab106.

Peixoto, Andreia, et al. "Protein Glycosylation and Tumor Microenvironment Alterations Driving Cancer Hallmarks." Frontiers in Oncology, vol. 0, Jan. 2019, https://doi.org/10.3389/fonc.2019.00380.

Santos, Mafalda, et al. "Codon Misreading TRNAs Promote Tumor Growth in Mice." RNA Biology, vol. 15, no. 6, 2018, pp. 773-86, https://doi.org/10.1080/15476286.2018.1454244.

Silver, Daniel P., and David M. Livingston. "Mechanisms of BRCA1 Tumor Suppression." Cancer Discovery, vol. 2, no. 8, Aug. 2012, pp. 679-84, https://doi.org/10.1158/2159-8290.CD-12-0221.

Singh, Nishant, and Anuradha Bharara Singh. "S2 Subunit of SARS-NCoV-2 Interacts with Tumor Suppressor Protein P53 and BRCA: An in Silico Study." Translational Oncology, vol. 13, no. 10, Oct. 2020, p. 100814, https://doi.org/10.1016/j.tranon.2020.100814.

Stingi, Aureliano, and Luca Cirillo. "SARS-CoV-2 Infection and Cancer: Evidence for and against a Role of SARS-CoV-2 in Cancer Onset." BioEssays : News and Reviews in Molecular, Cellular and Developmental Biology, vol. 43, no. 8, Aug. 2021, p. e2000289, https://doi.org/10.1002/bies.202000289.

(US), National Center for Biotechnology Information. "The P53 Tumor Suppressor Protein.” NCBI Bookshelf, 1 Jan. 1998, https://www.ncbi.nlm.nih.gov/books/NBK22268/.

Xia, Xuhua. "Detailed Dissection and Critical Evaluation of the Pfizer/BioNTech and Moderna MRNA Vaccines." Vaccines, vol. 9, no. 7, July 2021, p. 734, https://doi.org/10.3390/vaccines9070734. 